\title{
Die Auswirkungen des Corona-Shutdowns im März|April 2020: Der Zusammenhang zwischen Alltagsverhalten und psychischer Belastung bei psychisch Vorerkrankten und Gesunden
}

\section{Psychological Distress during the Corona Shutdown: Associations between Behavioral Adaption and Psychological Distress in Individuals with Preexisting Psychiatric Conditions and Healthy Controls}

\author{
Autoren \\ Daniel Nischk ${ }^{\circledR 1}$, Martin Voss ${ }^{2}$ \\ Institute \\ 1 Zentrum für Psychiatrie Reichenau \\ 2 Psychiatrische Universitätsklinik der Charité im St. Hedwig- \\ Krankenhaus
}

Schlüsselwörter

Corona, psychische Erkrankung, Alltagsverhalten, psychische Belastung

Key words

Corona, mental illness, daily activities

eingereicht 14.09 .2020

akzeptiert $\quad 29.12 .2020$

\section{Bibliografie}

Fortschr Neurol Psychiatr 2021; 89: 302-307

DOI 10.1055/a-1348-1242

ISSN 0720-4299

(C) 2021. Thieme. All rights reserved.

Georg Thieme Verlag KG, Rüdigerstraße 14,

70469 Stuttgart, Germany

Korrespondenzadresse

Daniel Nischk

Zentrum für Psychiatrie Reichenau

Feursteinstr. 55, 78479 Reichenau

Germany

Tel.: +4975319778761

Fax: +497531977567

E-Mail: d.nischk@zfp-reichenau.de

\section{ZUSAMMENFASSUNG}

Ziel Menschen mit psychischen Vorerkrankungen scheinen nach ersten Studienergebnissen aus anderen Ländern durch den Corona-Shutdown im Frühjahr 2020 besonders psychisch belastet gewesen zu sein. Um für etwaige künftige Krisen angemessene Versorgungsstrukturen vorhalten zu können, wurde in dieser Studie die psychische Belastung psychisch Vorerkrankter mit der psychisch
Gesunder während des Shutdowns verglichen und darüber hinaus der Zusammenhang zwischen Alltagsverhalten und psychischer Belastung untersucht.

Methodik In einer 15-minütigen Online-Befragung wurden 44 Menschen mit stationären psychiatrischen Vorbehandlungen und 55 psychisch Gesunde hinsichtlich ihrer generellen psychischen Belastung und der Veränderungen ihres Alltagsverhaltens seit Beginn der Einschränkungen befragt.

Ergebnisse Menschen mit psychischen Vorerkrankungen waren insgesamt signifikant stärker psychisch belastet als Gesunde ( $<<0,001 ; d=1,68)$ und berichteten auch über signifikant geringere konstruktive Anpassungen ihres Alltagsverhaltens $(p=0,012 ; d=-0,52)$ an die veränderte Lebenssituation. 20,6\% der höheren psychischen Belastung ließ sich statistisch durch die geringere Anpassung des Alltagsverhaltens erklären.

Schlussfolgerungen Die Befunde legen nahe, dass psychisch vorerkrankte Menschen gerade in Krisenzeiten ein differenziertes sozialpsychiatrisches Angebot benötigen, welches sie im Alltag unterstützt, um notwendige Anpassungen des Alltagslebens an eine dramatisch veränderte Umgebung besser meistern zu können.

\section{ABSTRACT}

Objective Current research suggests that individuals with preexisting psychiatric conditions experienced particularly high levels of psychological distress during the various 'shutdown' measures to contain Covid-19. In order to gain a better insight into the demands for psychiatric care in times of crisis, this study compared levels of psychological distress in individuals with preexisting psychiatric conditions with healthy controls and further examined associations of daily routines with psychological distress. Method: Out of 99 participants of an online survey, 44 individuals reported prior mental health-related inpatient treatment. Patients were asked about their levels of psychological distress and adaptation of lifestyle and activities of daily living.

Results Individuals with a psychiatric history were significantly more psychologically distressed $(p<0.001$; $d=1.68)$ 
and displayed significantly less behavioral adaptation than healthy controls $(p=0.012 ; d=-0.52$ ) in response to the changed circumstances. The difference in behavioral adaptation accounted for $21 \%$ of the difference in psychological distress.
Conclusions: In times of crisis, individuals with a psychiatric history require ongoing support from mental health services, in particular those supporting every-day lifestyle in order to better cope with the consequences of a drastically changed environment.

\section{Einleitung}

Die zur Eindämmung der Corona-Pandemie ergriffenen Maßnahmen haben in Deutschland zwischen Mitte März und Anfang Mai 2020 das öffentliche Leben der Allgemeinheit und die private Freiheit des Einzelnen in einem nie dagewesenen Ausmaß beschränkt. Vorläufige Studienergebnisse aus verschiedenen Ländern weisen auf die erhebliche psychische Belastung während der Shutdown-Maßnahmen sowohl in der Allgemeinbevölkerung [13] als auch besonders bei Menschen mit psychischen Vorerkrankungen [4, 5] hin.

Dass psychisch Vorerkrankte besonders belastet auf Shutdown- und Quarantäne-Maßnahmen reagieren, entspricht den Erfahrungen aus anderen Pandemien [6] und scheint zumindest z.T. auch auf die unzureichende psychiatrische Versorgung während solcher Maßnahmen zurückzugehen [4, 5]. In Deutschland hatten viele psychiatrische Kliniken und Ambulanzen sowie zahlreiche Träger komplementärer Einrichtungen wie Kontakt- und Begegnungsstätten oder Suchtberatungsstellen infolge der Corona-Pandemie ihre Angebote zurückgefahren oder zeitweise ganz geschlossen.

Darüber hinaus scheinen insbesondere das Alltagsverhalten und der Lebensstil während Shutdown- oder Quarantäne-Maßnahmen bedeutsam [6]. Es ist bekannt, dass sich Alltagsverhalten und psychische Gesundheit wechselseitig beeinflussen können [7]. Die Corona-Krise hat allen Menschen größere Anpassungen im Alltagsverhalten in nahezu allen Lebensbereichen (Arbeit, Familie, Freizeit) abverlangt. Um gerade in solch belastenden Zeiten die psychische Gesundheit zu erhalten, sind neue Strategien, wie etwa Sport und Bewegung, alternative Formen der Kontaktpflege oder veränderte, an die neue Situation angepasste Tagesroutinen, nötig, wie auch Vorerfahrungen aus anderen Pandemien nahelegen [6]. Menschen mit psychischen Erkrankungen neigen jedoch ohnehin zu ungünstigem Alltagsverhalten [8, 9], was durch Erwerbslosigkeit, Mangel an sozialen Bezügen oder prekären Wohnverhältnissen (die z.B. durch Anonymität, räumliche Enge oder Wohnen in sozialen Brennpunkten gekennzeichnet sind) noch verschärft werden dürfte. Ein Schwerpunkt des umfangreichen stationären wie ambulanten (sozial-)psychiatrischen Angebotes zielt schon aus diesem Grund darauf ab, Menschen mit psychischen Erkrankungen bedarfsgerecht in praktischen Aspekten des Alltags und der Lebensführung zu unterstützen.

Vor diesem Hintergrund sind Erkenntnisse zum Ausmaß der Belastung psychisch Vorerkrankter sowie zum Alltagsverhaltens während des Shutdowns von immenser Bedeutung, da sie helfen können, in den sich bereits andeutenden zukünftigen Krisen angemessene Versorgungsangebote, etwa in Form von mehr aufsuchenden oder Online-Angeboten, vorzuhalten. Auch sollte der Gefahr, einzelne Versorgungsstrukturen vorschnell als „nicht notwendig“ einzustufen und möglicherweise ganz zu schließen, begegnet werden, zumal viele Hilfsangebote in der Krise auch deutlich weniger als zuvor genutzt wurden.

Um unter den auch für die Forschung eingeschränkten Bedingungen während der Corona-Krise zumindest erste Erkenntnisse zu den o.g. Aspekten zu gewinnen, führten wir während der Hochphase der Corona-Beschränkungen in Deutschland eine Online-Befragung durch, die den folgenden Fragen nachging:

1. Wie hoch ist die psychische Belastung bei Menschen mit psychischen Vorerkrankungen im Vergleich zu psychisch Gesunden? Wirkt sich die Corona-Krise unterschiedlich auf das Alltagsverhalten von psychisch Gesunden und Menschen mit psychischen Vorerkrankungen aus?

2. Welcher Zusammenhang besteht zwischen Veränderungen im Alltagsverhalten als Folge der Corona-Krise und psychischer Belastung bei psychisch Gesunden und Menschen mit psychischen Vorerkrankungen?

\section{Methodik}

\section{Procedere}

Teilnehmer wurden über die Website der Internationalen Arbeitsgemeinschaft Soteria (IAS; www.soteria-netzwerk.de) sowie über deren E-Mail-Newsletter rekrutiert. Die Website versorgt Menschen mit psychischen Erkrankungen, deren Angehörige, professionelle Helfer sowie Interessierte mit allgemeinen Informationen über das Soteria-Konzept, über die Tätigkeiten der Arbeitsgemeinschaft sowie über die einzelnen Einrichtungen. Die Datenerhebung fand zwischen dem 6.4. und 1.5.2020 in der Hochphase der Corona-Beschränkungen, statt. Die Teilnehmer wurden im Einklang mit der Helsinki-Deklaration über den Zweck der Befragung, über die Freiwilligkeit der Teilnahme sowie über die vollständige Anonymisierung der Daten aufgeklärt und darüber informiert, dass Ergebnisse über die Website zur Verfügung gestellt werden würden.

\section{Versuchspersonen}

Von 120 Probanden mussten 21 aufgrund von fehlenden Werten $>10 \%$ oder grob unplausiblen Antwortmustern ausgeschlossen werden, sodass 99 Datensätze zur Auswertung zur Verfügung standen. 44 Teilnehmer waren psychisch vorerkrankt (Kriterium: mindestens eine stationäre Vorbehandlung in einem psychiatrischen oder psychosomatischen Krankenhaus). 55 waren nach diesem Kriterium psychisch gesund ( $>$ Tab. 1). Hinsichtlich Geschlechtsverteilung, Bildungsabschluss und persönlicher Betroffenheit durch Covid-19 (z.B. eigene Infektion) waren die Gruppen vergleichbar, jedoch waren psychisch Vorerkrankte signifikant jünger $(42,43<48,00)$. Verschiedene Indikatoren zeigten bei den psychisch Vorerkrankten eine recht hohe Erkran- 
kungsschwere und psychosoziale Beeinträchtigung an: 26 $(59,1 \%)$ berichteten über mindestens zwei Diagnosen; 21 $(47,7 \%)$ waren bereits vor dem 21 . Lebensjahr erstmalig in psychiatrischer Behandlung. Darüber hinaus waren psychisch Vorerkrankte signifikant seltener beruflich beschäftigt (42,2\% vs. $18,2 \% ; p=0,007)$ und lebten signifikant seltener eigenständig (20,5\% vs. 1,8\%; p=0,005). 37 (84\%) erfüllten mindestens eines dieser Schweregrad-Kriterien; 25 (56,8\%) sogar mindestens zwei.

\section{Online-Befragung}

Die insgesamt ca. 15-minütige Online-Befragung wurde entsprechend der zum Zeitpunkt der Befragung verfügbaren Literatur zu anderen Pandemien [6] sowie klinischer Erfahrung zusammengestellt. Zunächst wurden demografische und klinische Basisdaten ( $\triangleright$ Tab. 1) erhoben, bevor nacheinander die folgenden Bereiche abgefragt wurden. Die psychische Belastung wurde anhand von 21 allgemeinen psychischen Symptomen (wie z.B. Ängsten, Reizbarkeit, Langeweile, Grübeln, Misstrauen oder Schlafstörungen) erhoben, deren Auftreten innerhalb der letzten 7 Tage anhand 4stufiger Likert-Skalen ( $0=$ „nicht vorhanden“ bis $3=$ „sehr stark“) eingeschätzt wurden. Darin enthalten waren 6 Items aus der Skala Depressivität aus der Brief Symptom Scale (BSI [10]), für die aus Normierungsstudien Vergleichswerte herangezogen werden konnten. Als Maß für die generelle psychische Belastung wurde der Gesamtmittelwert aller Items (bzw. der Subskala Depressivität) berechnet. Die innere Konsistenz der Gesamtskala war in der vorliegenden Studie mit Cronbachs Alpha $=0,95$ exzellent.

In den ersten 7 Tagen der Studie (6.4-13.4.2020) wurden die Teilnehmer gebeten, zusätzlich ihren Zustand vor Beginn der Corona-Einschränkungen (d.h. vor dem 18.3.2020) rückwirkend

-Tab. 1 Demografische, klinische Basisdaten sowie Befragungsergebnisse der Stichprobe ( $n=99)$.

\begin{tabular}{|c|c|c|c|}
\hline & $\begin{array}{l}\text { Psychisch Vorerkrankte } \\
(n=44)\end{array}$ & $\begin{array}{l}\text { Psychisch Gesunde } \\
(n=55)\end{array}$ & Teststatistik \\
\hline Alter & $42,43(13,66)$ & $48,00(11,61)$ & $t(97)=2,19 ; p=0,031$ \\
\hline Alter Erstbehandlung & $24,14(10,34)$ & - & - \\
\hline Weiblich & $28(63,6)$ & $40(72,7)$ & $X^{2}(1)=7.38 ; p=0,939$ \\
\hline \multicolumn{4}{|l|}{ Selbstberichtete } \\
\hline \multicolumn{4}{|l|}{ Diagnosen } \\
\hline Psychose & $14(31,8)$ & & \\
\hline Affektive Störung & $33(75,0)$ & & \\
\hline Sucht & $8(18,2)$ & & \\
\hline Persönlichkeitsst. & $11(25,0)$ & & \\
\hline 1 Diagnose & $18(40,9)$ & & \\
\hline$\geq 2$ Diagnosen & $26(59,1)$ & & \\
\hline $\begin{array}{l}\text { Vor dem 21. Lebensjahr erstmals } \\
\text { in Behandlung }\end{array}$ & $21(47,7)$ & & \\
\hline Abitur/Studium & $30(68,2)$ & $45(81,8)$ & $X^{2}(1)=2,47 ; p=0,116$ \\
\hline Nicht selbstständiges Wohnen ${ }^{a}$ & $9(20,5)$ & $1(1,8)$ & $\begin{array}{l}\text { Fisher's Exact Test: } \\
p=0,005\end{array}$ \\
\hline Nicht erwerbstätig & $19(43,2)$ & $10(18,2)$ & $X^{2}(1)=7,38 ; p=0,007$ \\
\hline $\begin{array}{l}\text { Persönliche Betroffenheit durch } \\
\text { Covid- } 19^{\text {b }}\end{array}$ & $8(18,2)$ & $6(10,9 \%)$ & $X^{2}(1)=1,07 ; p=0,39$ \\
\hline Symptombelastung & $1,17(0,67)$ & $0,16(0,54)$ & $t(81,68)=-0,49$ \\
\hline \multirow[t]{2}{*}{ Vorher vs. jetzt ${ }^{c}$} & $2,04(0,58)$ vs. & - & $p=<0,001$ \\
\hline & $2,24(0,69)$ & & $t(22)=-1,98 ; p=0,060$ \\
\hline \multirow[t]{3}{*}{ Depressivität $^{d}$} & $1,13(0,86)$ & $0,45(0,48)$ & \\
\hline & & & $U=766 ; Z=-3,89$ \\
\hline & & & $p<0,001$ \\
\hline $\begin{array}{l}\text { Anpassung im funktionalen } \\
\text { Alltagsverhalten }^{\text {e }}\end{array}$ & $-0,03(0,80)$ & $0,38(0,79)$ & $t(97)=2,56 ; p=0,012$ \\
\hline
\end{tabular}


anhand derselben Items einzuschätzen. Bei 23 psychisch Vorerkrankten konnte ein Prä-/Post-Vergleich durchgeführt werden. Bei den psychisch Gesunden war die Stichprobe zu klein für einen solchen Vergleich.

Die Veränderungen im Alltagsverhalten wurden durch 14 Items ( $\triangleright$ Abb. 1) auf einer Skala zwischen -3 (= „viel seltener“) bis +3 (= „viel häufiger“) sowie „nicht zutreffend“ (als Ausschlusskategorie) erhoben. Für die weitere Analyse wurden negativ formulierte Items umgepolt und zu einer Skala Veränderungen im funktionalen Alltagsverhalten zusammengefasst. Die interne Konsistenz dieser Skala war mit Cronbachs Alpha = 0,85 zufriedenstellend.

Persönliche Betroffenheit durch Covid-19 wurde kodiert (Nein = 0 ; Ja =1), sofern der Teilnehmer von persönlicher Quarantäne, Covid-19-Infektion oder Erkrankung, Jobverlust, Erkrankung im familiären oder näheren sozialen Umfeld betroffen war.

\section{Datenaufbereitung und Auswertung}

Die Auswertung erfolgte mithilfe von SPSS (Version 23) und dem Plugin Process [11]. Die einzelnen Items bzw. Skalen wurden ebenso wie einzelne Datenreihen explorativ u.a. durch geeignete deskriptive Maße, Zusammenhangsmaße und Trennschärfekoeffizienten sorgfältig auf Konsistenz und Plausibilität überprüft und einzelne Items bzw. Teilnehmer ausgeschlossen. Unterschiede wurden mittels t-Tests (bzw. U-Test als nonparametrische Alternative) oder $\mathrm{X}^{2}$ Test (bzw. Fisher's Exact Test) überprüft. Das Signifikanzniveau wurde auf Alpha=0,05 gesetzt. Bei multiplen Vergleichen wurde das Alpha-Niveau zusätzlich nach Benjamini und Hochberg korrigiert. Da bei kleinen Stichproben hierdurch auch tatsächliche

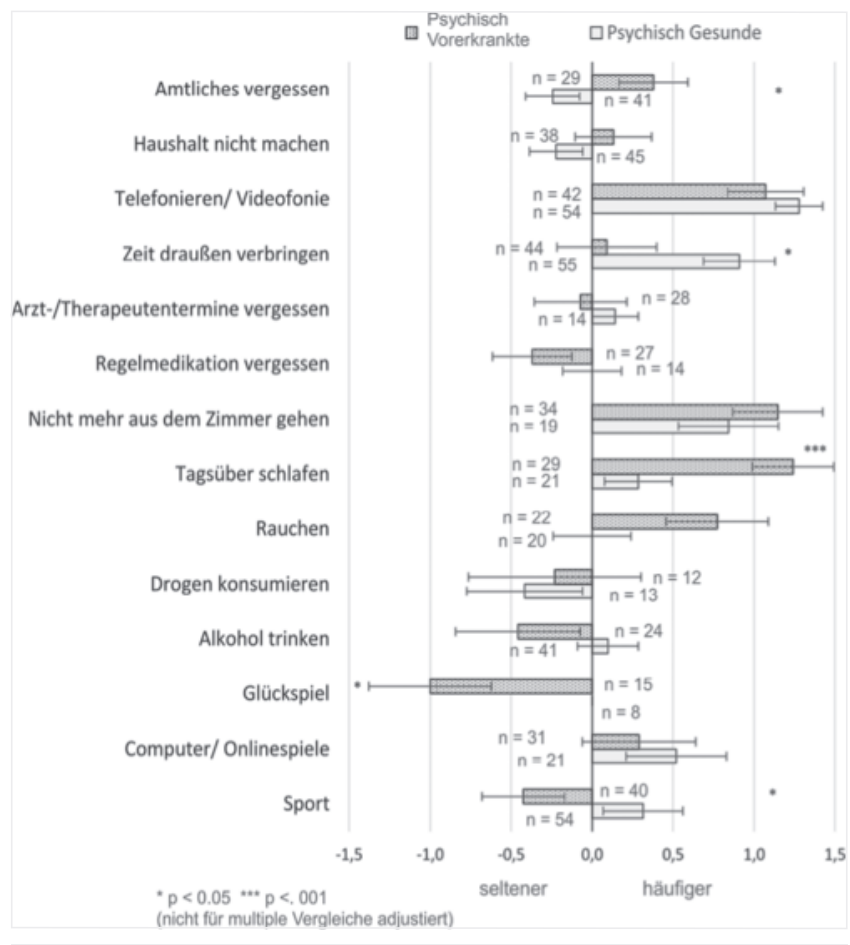

Abb. 1 Veränderungen im Alltagsverhalten aufgrund der CoronaKrise bei psychisch Gesunden $(n=55)$ und bei psychisch Vorerkrankten $(n=44)$. Angegeben sind Mittelwerte und Standardfehler sowie die Anzahl der Teilnehmer, auf die das Item zutraf.
Unterschiede verschwinden können, werden sowohl korrigierte wie auch nichtkorrigierte Ergebnisse angegeben. Als Effektstärke wurde Cohens d (mit entsprechender Korrektur bei unterschiedlicher Stichprobengröße) herangezogen (0,2 = „klein“, 0,5= „mittel“, $0,8=$ „groß“ [2]). Zusammenhänge wurden als Pearson-Korrelation (bzw. als Punkt-Biseriale Korrelation) dargestellt.

Die Veränderungen des funktionalen Alltagsverhaltens wurde als mediierender Faktor zwischen Gruppe $(0=$ psychisch gesund; 1 = psychisch krank) und psychischer Belastung definiert und entsprechend durch Regressionsanalysen überprüft. Da sich die Gruppen hinsichtlich verschiedener Merkmale unterschieden, wurde abschließend explorativ die Stabilität der Modellparameter für verschiedene Subgruppen überprüft. Hierzu wurden die Variablen gegenwärtige Berufstätigkeit, unabhängiges Wohnen und Alter > 40 jeweils einzeln als „dummy“-kodierte Kovariate $(0=$ „nein“, 1 = , ja“) in das Mediationsmodell eingefügt.

Die Voraussetzungen für die angegebenen Verfahren waren ausreichend erfüllt.

\section{Ergebnisse}

Die Symptombelastung war bei psychisch Vorerkrankten insgesamt deutlich und signifikant höher als bei Gesunden (1,17>0,16; $p<0,001 ; d=1,68 ; \triangleright$ Tab. 1). Auf 19 der 21 Items zeigten psychisch Vorerkrankte auch nach Korrektur für multiples Testen signifikant höhere Werte (alle p > 0,05). Auf der Skala Depressivität des BSI wiesen psychisch Vorerkrankte in Bezug auf die Normstichprobe (600 psychisch Gesunden [10]) einen Prozentrang von 94 auf (d. h., $94 \%$ der Normstichprobe erzielten niedrigere oder gleiche Werte), während psychisch Gesunde - immer noch deutlich erhöht - bei Prozentrang 71 lagen. Ergänzende Informationen zur symptomatischen Belastung sind auch auf der Webseite der Internationalen Arbeitsgemeinschaft Soteria (soteria-netzwerk.de) verfügbar. Bei 23 (von 44) psychisch Vorerkrankten lagen auch rückwirkende Einschätzungen aus der Zeit vor den Corona-Einschränkungen vor: Die Symptombelastung erhöhte sich demnach in der Zeit der Corona-Einschränkungen (2,24 vs. 2,04), was einem starken Trend entsprach $(p=0,060)$. In der Gruppe der Gesunden lagen nicht genug Prä-/Post-Einschätzungen für einen solchen Vergleich vor.

Über beide Gruppen war die Symptombelastung negativ mit unabhängigem Wohnen ( $r=-0,39 ; p<0,001)$, gegenwärtiger Tätigkeit $(r=-0,24 ; p=0,017)$ sowie Alter $(r=-0,43 ; p<0,001)$ korreliert.

Psychisch Gesunde gaben signifikante Veränderungen im konstruktiven Alltagsverhalten ( $\triangleright$ Tab. 1) als Folge der Corona-Krise an $(0,38 ; p=0,001)$, nicht jedoch psychisch Vorerkrankte $(-0,02$; $\mathrm{p}=0,889$ ). Der Unterschied in der Anpassung im konstruktiven Alltagsverhalten zwischen psychisch Gesunden und psychisch Vorerkrankten war signifikant ( $p=0,012 ; d=-0,52$, $>$ Abb. 1). Signifikante Unterschiede zugunsten der psychisch Gesunden ergaben sich bei Sporttreiben ( $p=0,043 ; d=-0,44)$, tagsüber schlafen $(p=0,005 ; d=0,79)$, Zeit draußen verbringen $(p=0,029$; $d=-0,45)$ und Amtliches aufschieben $(p=0,023 ; d=0,56)$. Lediglich im Bereich Glücksspiel zeigten psychisch Kranke ein signifikant günstigeres Verhalten ( $p=0,019 ; p=-0,84)$. Bei Anwendung der Benjamini-Hochberg-Korrektur für multiples Testen waren jedoch alle Unterschiede nur noch als Trend vorhanden. 


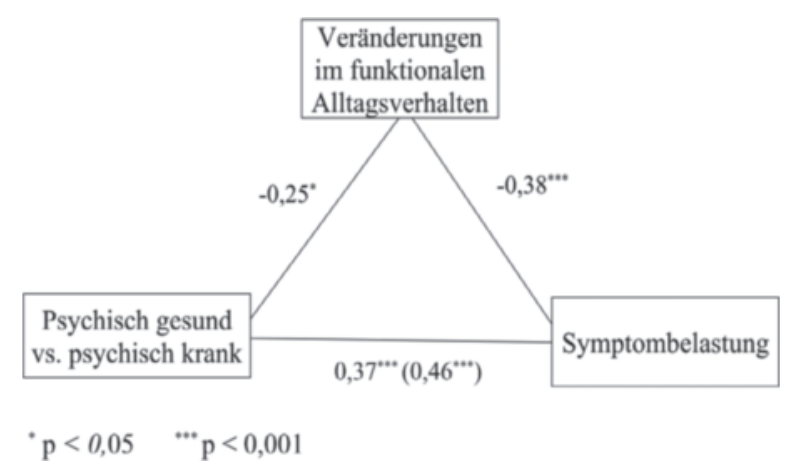

-Abb. 2 Funktionales Alltagsverhalten mediiert den Zusammenhang zwischen Gruppenzugehörigkeit (psychisch gesund vs. psychisch vorerkrankt) und Symptombelastung. Alle Werte sind standardisiert ( $\beta$ ). In Klammern ist der Zusammenhang ohne den Mediationsterm angegeben. Die einzelnen Items der Skala Änderung des funktionalen Alltagsverhaltens wurden so umkodiert, dass höhere Werte auf häufigeres günstiges Verhalten hinweist.

Die Veränderungen im funktionalen Alltagsverhalten übten einen mediierenden Einfluss auf die Symptombelastung aus ( Abb. 2): Psychisch Vorerkrankte berichteten über geringere Veränderungen im funktionalen Alltagsverhalten $(\beta=-0,25$; $p=0,012$ ) als Gesunde. Positive Veränderungen im funktionalen Alltagsverhalten gingen hingegen mit geringerer Symptombelastung einher $(\beta=-0,33 ; p<0,001)$. Der indirekte (standardisierte) Effekt auf die Symptombelastung $(-0,25 \times-0,33=0,095)$ entsprach $20,6 \%$ des Gesamteffektes $(\beta=0,46)$. Rund $21 \%$ des Unterschieds bei der psychischen Belastung zwischen psychisch Gesunden und psychisch Vorerkrankten gingen demnach statistisch auf die geringere Anpassung des funktionalen Alltagsverhaltens zurück. Sämtliche Ergebnisparameter des Mediationsmodells blieben auch dann signifikant, wenn dichotome Subgruppen ( 0 = nein, $1=$ ja) in Bezug auf gegenwärtige berufliche Tätigkeit, nichtselbstständigem Wohnen und Alter $>40$ gebildet wurden und jeweils einzeln als Kovariate in das Mediationsmodell eingefügt wurden. Aufgrund der geringen Stichprobengrößen [range 8-40] sind diese Ergebnisse jedoch nur eingeschränkt aussagekräftig.

\section{Diskussion}

In dieser Online-Befragung wurde eine Gruppe psychisch Vorerkrankter mit recht hohem Schweregrad mit psychisch Gesunden verglichen. Die Ergebnisse deuten auf eine erheblich höhere psychische Belastung von psychisch Vorerkrankten im Vergleich zu psychisch Gesunden hin. Die erhöhte psychische Belastung stellt mit hoher Wahrscheinlichkeit eine Auswirkung der Corona-Krise dar, wie der Prä/Postvergleich bei einer Teilstichprobe von 23 psychisch Vorerkrankten nahelegt, die während der ersten 7 Tage der Befragung ihre psychische Belastung rückwirkend einschätzten. Die Symptombelastung bei den psychisch Vorerkrankten war auf der Skala Depressivität mit einem mittleren Prozentrang von 94 erheblich, bei psychisch Gesunden jedoch immer noch erhöht (Prozentrang 71). Die Ergebnisse replizieren die jüngst publizierten Befunde aus China und Italien [4, 5], die ebenfalls eine höhere psychische Belastung bei psychisch Vorerkrankten infolge der Corona-Pandemie nahelegen.

Während psychisch Gesunde jedoch ihr Alltagsverhalten konstruktiv auf die veränderten Umstände anpassten, war dies bei den psychisch Vorerkrankten kaum der Fall. Zwar ließen sich die Unterschiede (nach Korrektur für multiples Testen) nicht auf bestimmte Verhaltensmuster zurückführen, jedoch mediierte der gemittelte Gesamtunterschied in den Veränderungen im konstruktiven Alltagsverhalten die Symptombelastung signifikant. Immerhin $21 \%$ der Unterschiede in der Symptombelastung zwischen psychisch Gesunden und psychisch Vorerkrankten gingen statistisch auf diesen indirekten Effekt zurück. Das Gesamtmuster der Ergebnisse blieb auch dann bestehen, wenn jeweils einzeln gegenwärtige Berufstätigkeit, nichtselbstständiges Wohnen, Alter > 40 und Geschlecht statistisch berücksichtigt wurden, wobei diese Ergebnisse aufgrund der geringen Größe der Teilstichproben nicht überbewertet werden sollten.

Unser Ergebnis zeigt in Ergänzung zu Befunden, die ein oft dysfunktionales Alltagsverhalten bei psychisch Kranken im Vergleich zu Gesunden zeigen [5, 6], dass sich psychisch Kranke auch kaum oder weniger adaptiv auf die Umstände der CoronaKrise einstellen konnten. Die deutlich geringer ausgeprägte Anpassung an veränderte Umstände im Alltag, wie Sport treiben, trotz verringerter äußerer „Taktgeber“ einen Tagesrhythmus beibehalten, regelmäßiger Aufenthalt im Freien oder das prompte Erledigen wichtiger Tätigkeiten, deuten auf einen stärkeren sozialen Rückzug und depressive Verhaltensmuster hin. In welchem Ausmaß dieses verminderte Anpassungsverhalten durch bedarfsgerechte Hilfsangebote auch während eines Corona-Shutdowns verhindert werden könnte, bleibt jedoch spekulativ. Schließlich muss angemerkt werden, dass die Ergebnisse zwar den im Mediationsmodell postulierten Einfluss des Alltagsverhaltens auf die Symptombelastung bestätigen, jedoch ist zusätzlich die umgekehrte Wirkrichtung, von der Symptombelastung auf das Alltagsverhalten, nicht nur theoretisch plausibel [7], sondern aufgrund des querschnittlichen Designs auch möglich. Dies eröffnet weitere therapeutische Möglichkeiten über die Unterstützung im Alltag hinaus, etwa medikamentöse Anpassungen oder therapeutische Gespräche. Jedenfalls scheint klar, dass in CoronaZeiten die Strukturvorgaben des psychiatrischen Hilfesystems u. a. in Richtung aufsuchende Hilfe („Walk \& talk“-Angebote) und digitaler Unterstützung (z.B. Videofonie) erweitert werden müssen. Erste publizierte Erfahrungen [12] deuten darauf hin, dass die Corona-Krise durchaus auch Chancen bietet, bereits eingeleitete notwendige sozialpsychiatrische Entwicklungen, z.B. von der Mentalität der Versorgung zu der des Empowerments, aufzugreifen und zu forcieren.

Es scheint daher unstrittig, und die Ergebnisse dieser Studie unterstreichen dies, dass auch und gerade in Krisenzeiten psychisch Kranke ein gemeindenahes sozialpsychiatrisches Angebot benötigen, das sie insbesondere im Alltag unterstützt. Das Fernbleiben von verfügbaren Angeboten scheint eher die Folgen der erhöhten psychischen Belastung anzuzeigen - nicht den fehlenden Bedarf. 


\section{Limitationen}

Die Ergebnisse unserer Studie können aufgrund der kleinen, selektiv über die Soteria-Website bzw. den Soteria-Newsletter aggregierten Stichprobe natürlich nicht als repräsentativ gelten: Bei den psychisch Vorerkrankten mögen z.B. negative Erfahrungen mit der Psychiatrie überrepräsentiert sein. Die Gruppe der psychisch Gesunden könnte zu einem hohen Anteil aus Angehörigen und Mitarbeitern aus psychiatrischen Institutionen bestehen.

Ferner muss bei den vorliegenden, hinsichtlich verschiedener demografischer Merkmale nicht ausbalancierten Stichproben auch offen bleiben, in welchem Ausmaß die Unterschiede bei der psychischen Belastung tatsächlich lediglich auf den Umstand der psychischen Vorerkrankung zurückzuführen sind oder ggf. auch durch zusätzliche Arbeitslosigkeit, nicht eigenständiges Wohnen oder jüngeres Lebensalter verstärkt wurden. Die vorliegende, noch sehr vorläufige Forschungslage deutet darauf hin, dass Merkmale sozialer Benachteiligung (z.B. geringes Einkommen, Minderheitenstatus oder alleiniges Wohnen) neben einem Lebensalter unter 25 [1-5] auch für sich genommen mit höherer psychischer Belastung während der Corona-Krise assoziiert waren. Da die psychisch Vorerkrankten ebenfalls recht hoch psychosozial beeinträchtigt waren, mag dies einen Teil ihrer erhöhten Belastung erklären. Dass das Ergebnismuster im Mediationsmodell auch bei der statistischen Berücksichtigung weiterer Faktoren stabil blieb, zeigt in Ergänzung zu einer jüngst publizierten Studie aus Italien [5], dass mit hoher Wahrscheinlichkeit bereits der Umstand einer psychischen Vorerkrankung an sich mit höherer Belastung assoziiert ist. Darüber hinaus erheben Online-Befragungen natürlich lediglich subjektive Daten, deren Validität letztlich nur abgeschätzt werden kann. Die rückwirkende Einschätzung der subjektiven psychischen Befindlichkeit in der Befragung kann überdies Gedächtnisverzerrungen unterliegen.

Allen Limitationen zum Trotz können die vorliegenden Daten einen ersten Einblick in ein bislang wenig untersuchtes Themenfeld geben und zu weiteren Studien ermutigen.

\section{Konsequenzen für Klinik und Praxis}

- Menschen mit psychischen Vorerkrankungen waren durch die Shutdown- und Quarantänemaßnahmen im März dieses Jahres besonders stark psychisch belastet.

- Ein Teil dieser psychischen Belastung ging auf die mangelnde Anpassung des Alltagsverhaltens an die dramatisch veränderten Lebensumstände zurück.
- Für etwaige künftige Krisen sollten daher insbesondere die Entwicklung aufsuchender und digitaler Versorgungsangebote vorangetrieben werden, die Alltagsverhalten und Symptombelastung in den Blick nehmen.

\section{Interessenkonflikt}

Die Autorinnen/Autoren geben an, dass kein Interessenkonflikt besteht.

Literatur

[1] Wang C, Pan R, Wan X et al. Immediate psychological responses and associated factors during the initial stage of the 2019 coronavirus disease (COVID-19) epidemic among the general population in China. Int J Environ Res Public Heal 2020; 17: 1729

[2] Pierce M, Hope $\mathrm{H}$, Ford $\mathrm{T}$ et al. Mental health before and during the COVID-19 pandemic: A longitudinal probability sample survey of the UK population. Lancet Psychiatry 2020; 0366: 1-10

[3] McGinty EE, Presskreischer R, Han $\mathrm{H}$ et al. Psychological distress and loneliness reported by US adults in 2018 and april 2020. JAMA - J Am Med Assoc 2020; 324: 93-94

[4] Zhou J, Liu L, Xue P et al. Mental health response to the COVID-19 outbreak in China. Am J Psychiatry 2020; 177: 574-575

[5] lasevoli F, Fornaro M, D'Urso G et al. Psychological distress in patients with serious mental illness during the COVID-19 outbreak and onemonth mass quarantine in Italy [published online ahead of print, 2020 May 19]. Psychol Med. 2020; 1-3. doi:10.1017/S0033291720001841

[6] Brooks SK, Webster RK, Smith LE et al. The psychological impact of quarantine and how to reduce it: Rapid review of the evidence. Lancet 2020; 395: 912-920

[7] Velten J, Lavallee KL, Scholten $S$ et al. Lifestyle choices and mental health: A representative population survey. BMC Psychol 2014; 2: 1-11

[8] Ritsner M, Ben-Avi I, Ponizovsky A et al. Quality of life and coping with schizophrenia symptoms. Qual Life Res 2003; 12: 1-9

[9] Meyer B. Coping with severe mental illness: Relations of the brief COPE with symptoms, functioning, and well-being. C] Psychopathol Behav Assess 2001; 23: 265-277

[10] Franke GH. BSI: Brief Symptom Inventory von LR Derogatis:(Kurzform der SCL-90-R): Deutsche Version: Testmappe. Beltz 2000

[11] Hayes AF. PROCESS: A versatile computational tool for observed variable medation, moderation, and conditional process modelling. 2012; Im Internet: www.afhayes.com/public/process2012.pdf

[12] Kreß H. Theißing A: "Psychiatrische Rehabilitation in Zeiten von Corona - Zwischen Systemrelevanz und Betretungsverbot. Sozialpsychiatr Informatonen 2020; 4: 39-41 